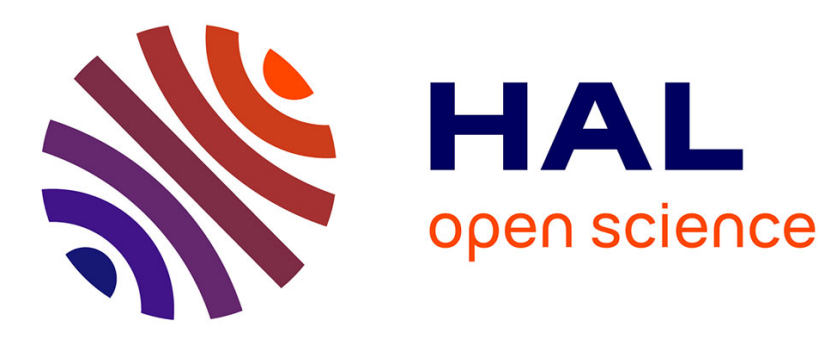

\title{
Texturation de YBa2Cu3O7-x par la méthode de l'échangeur thermique
}

\author{
A. Oçafrain, J. Chaminade, P. Dordor, L. Rabardel, M. Pouchard
}

\section{To cite this version:}

A. Oçafrain, J. Chaminade, P. Dordor, L. Rabardel, M. Pouchard. Texturation de YBa2Cu3O7x par la méthode de l'échangeur thermique. Journal de Physique III, 1994, 4 (11), pp.2131-2136. 10.1051/jp3:1994264 . jpa-00249248

\section{HAL Id: jpa-00249248 https://hal.science/jpa-00249248}

Submitted on 1 Jan 1994

HAL is a multi-disciplinary open access archive for the deposit and dissemination of scientific research documents, whether they are published or not. The documents may come from teaching and research institutions in France or abroad, or from public or private research centers.
L'archive ouverte pluridisciplinaire HAL, est destinée au dépôt et à la diffusion de documents scientifiques de niveau recherche, publiés ou non, émanant des établissements d'enseignement et de recherche français ou étrangers, des laboratoires publics ou privés. 


\title{
Texturation de $\mathrm{YBa}_{2} \mathrm{Cu}_{3} \mathrm{O}_{7-x}$ par la méthode de l'échangeur thermique
}

\author{
A. Oçafrain, J. P. Chaminade, P. Dordor, L. Rabardel et M. Pouchard \\ Laboratoire de Chimie du Solide, Université de Bordeaux 1, 351 cours de la Libération, \\ 33405 Talence Cedex, France
}

(Reçu le 3 février 1994, révisé le 12 avril 1994, accepté le 6 mai 1994)

\begin{abstract}
Résumé. - Un dispositif de croissance cristalline basé sur la technique de l'échangeur thermıque a été développé au laboratoire. Cette méthode a été appliquée à l'obtention de céramiques orientées de $\mathrm{YBa}_{2} \mathrm{Cu}_{3} \mathrm{O}_{7,}$. Les conditions expérimentales ainsı que les différents paramètres thermiques afférents à la méthode de texturation utilisée sont précisés. La texturation des échantıllons obtenus a été caractérisée par diffractıon des rayons $X$, par microscopie optıque et par microscopie électronique à balayage : la susceptibilité alternatıve a révélé une transition supraconductrice à $92 \mathrm{~K}$ et une densité de courant critique de $5000 \mathrm{~A} / \mathrm{cm}^{2}$ à $77 \mathrm{~K}$ et sans champ magnétique.

Abstract. - The Heat Exchanger Method (HEM) has been used to prepare bulk textured $\mathrm{YBa}_{2} \mathrm{Cu}_{3} \mathrm{O}_{7-1}$, ceramics. The experimental process and the thermal parameters used for texturing are described. The resulting bulk textured ceramics have been investigated by $\mathrm{X}$-ray diffraction, optical and scanning electronic microscopies. A $92 \mathrm{~K}$ temperature transition and a $J_{\mathrm{c}}$ of $5000 \mathrm{~A} / \mathrm{cm}^{-}$at $77 \mathrm{~K}$ in the absence of magnetic field have been shown by ac susceptibility measurements.
\end{abstract}

\section{Introduction.}

Un paramètre essentiel pour obtenir des densités de courant critique élevées dans $\mathrm{YBa}_{2} \mathrm{Cu}_{3} \mathrm{O}_{7-1}$, sous forme massive est la présence d'une microstructure texturée de manière à ce que les directions de haute densité de courant critique dans le plan basal soient alignées dans le sens de circulation du courant.

Actuellement les techniques de solidification directionnelle sont très utilisées pour produire des échantillons d' $\mathrm{YBa}_{2} \mathrm{Cu}_{3} \mathrm{O}_{7,}$, massifs avec une densité de courant critique supérieure à $10000 \mathrm{~A} / \mathrm{cm}^{2}$ dans des champs appliqués de plusieurs teslạs.

Bien que les variantes soient nombreuses dans les techniques de texturation par voie liquide, elles comportent toutes le chauffage de $\mathrm{YBa}_{2} \mathrm{Cu}_{3} \mathrm{O}_{7-1}$ au-dessus de la température de décomposition péritectique $\left(1014^{\circ} \mathrm{C}\right.$ à l'air) suivi d'un refroidissement lent de manière à former des grains de $\mathrm{YBa}_{2} \mathrm{Cu}_{3} \mathrm{O}_{7-1}$ alignés et généralement larges. 
La méthode HEM (Heat Exchanger Method) constituant une technique de solidification directionnelle, son application à la texturation de $\mathrm{YBa}_{2} \mathrm{Cu}_{3} \mathrm{O}_{7-1}$ a été entreprise. Les premiers résultats ont conduit à des céramiques texturées mais non optimisées.

Ce travail rend compte de la méthode de préparation de ces céramiques et de leur caractérisation: microstructure et propriétés supraconductrices.

\section{Méthode de l'échangeur thermique (HEM).}

La technique HEM est un processus simple de solidification directionnelle permettant de produire des lingots monocristallins de grande dimension (jusqu'à $30 \mathrm{~cm}$ de diamètre pour des cristaux de saphir [1]) et de haute qualité. Un schéma d'ensemble du four est représenté à la figure 1 .

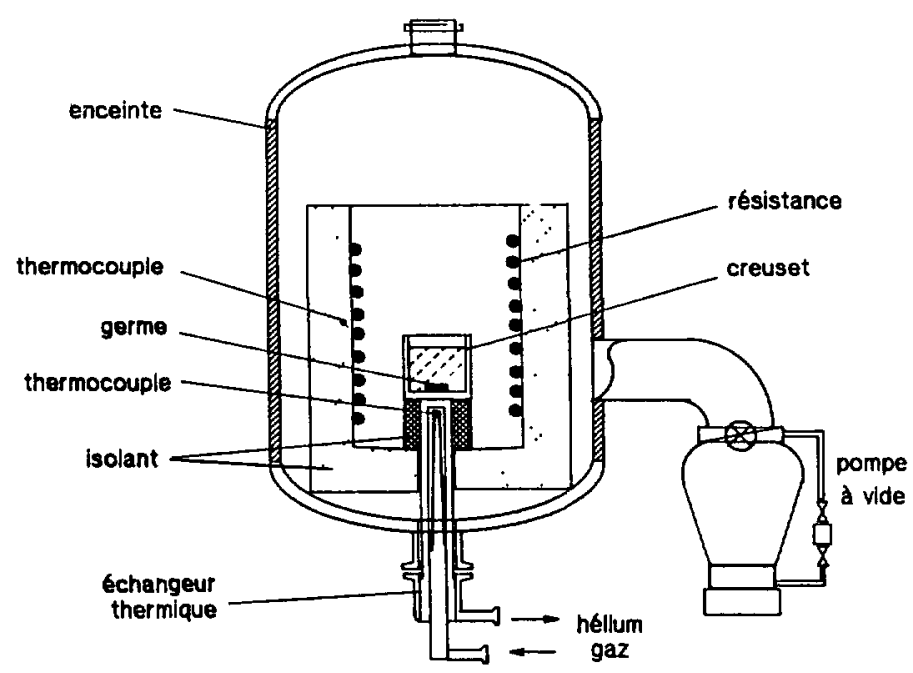

Fig. 1. - Schéma du four HEM.

[Schematic of the HEM furnace.]

La croissance cristalline est généralement initiée à partir d'un germe d'orientation déterminée, placé au bas du creuset. Le creuset rempli avec la charge est alors positionné sur l'échangeur thermique dans le four HEM. L'enceinte double paroi du four, refroidie à l'eau, permet d'opérer sous vide, à l'air ou sous atmosphère contrôlée.

La charge est portée à fusion par élévation de la température du four; le germe est maintenu à l'état solide en faisant circuler de l'hélium gazeux dans l'échangeur thermique.

Une fois le bain stabilisé, la croissance cristalline est réalisée en pilotant à la fois la descente en température du four et l'extraction de la chaleur par l'échangeur.

Les températures du four et de l'échangeur peuvent être modifiées au cours de la croissance.

Lorsque la solidification est accomplie, le lingot est encore dans la zone chaude et, par diminution du débit d'hélium, les gradients de température peuvent être réduits pour un recuit in situ, suivi d'un refroidissement contrôlé de l'ensemble.

Contrairement à beaucoup d'autres techniques de croissance qui nécessitent la création d'un gradient de température par déplacement du creuset, du cristal ou de la zone chaude, la technique HEM ne présente aucune pièce mobile. De plus elle comporte des gradients de température dans le liquide et dans le solide indépendants ; ceux-ci peuvent être modifiés aux différentes étapes du cycle de croissance. 


\section{Procédure expérimentale.}

La poudre de départ (lot de poudre $\mathrm{YBa}_{2} \mathrm{Cu}_{3} \mathrm{O}_{7-x}$ fournie par Rhône-Poulenc) est d'abord calcinée à l'air, à $870^{\circ} \mathrm{C}$ pendant $48 \mathrm{~h}$, puis à $930^{\circ} \mathrm{C}$ pendant $96 \mathrm{~h}$, en creuset d'alumine. Celui-ci est ensuite placé sur l'échangeur thermique dans le four HEM.

La texturation est réalisée ici sans germe.

Le cycle thermique, effectué à l'air, se déroule en plusieurs étapes :

- le four est d'abord porté à $1100^{\circ} \mathrm{C}$; cette température est nécessaire pour obtenir la décomposition péritectique ( $1014^{\circ} \mathrm{C}$ à l'air) dans toute la masse, compte tenu du fort gradient axial inhérent à la technique utilisée, et ce malgré l'absence de refroidissement par l'échangeur. La décomposition de $\mathrm{YBa}_{2} \mathrm{Cu}_{3} \mathrm{O}_{7}$, conduit à la formation de la phase $\mathrm{Y}_{2} \mathrm{BaCuO}_{5}$ et d'une phase liquide riche en baryum et en cuivre ;

- la cinétique de la réaction péritectique

$$
\mathrm{Y}_{2} \mathrm{BaCuO}_{5}+\mathrm{liq} \rightarrow \mathrm{YBa}_{2} \mathrm{Cu}_{3} \mathrm{O}_{7-1}
$$

étant lente, la cristallisation doit s'effectuer par un refroidissement très lent de l'échantillon [2].

Une relation liant la température imposée à l'échangeur thermique et la température effective au sein du produit a été établie ; ainsi, la partie inférieure de la masse est refroidie à $1^{\circ} \mathrm{C} / \mathrm{h}$ de $1020^{\circ} \mathrm{C}$ à $990^{\circ} \mathrm{C}$, puis à $4{ }^{\circ} \mathrm{C} / \mathrm{h}$ de $990^{\circ} \mathrm{C}$ à $850^{\circ} \mathrm{C}$.

Les évolutions thermiques lors du traitement de texturation sont représentées à la figure 2 ;

- les lingots obtenus subissent ultérieurement un recuit sous balayage d'oxygène (palier à $550^{\circ} \mathrm{C}$ puis refroidissement lent à $2{ }^{\circ} \mathrm{C} / \mathrm{h}$ de $550^{\circ} \mathrm{C}$ à $320^{\circ} \mathrm{C}$ ), ceci afin d'optimiser la teneur en oxygène du matériau.

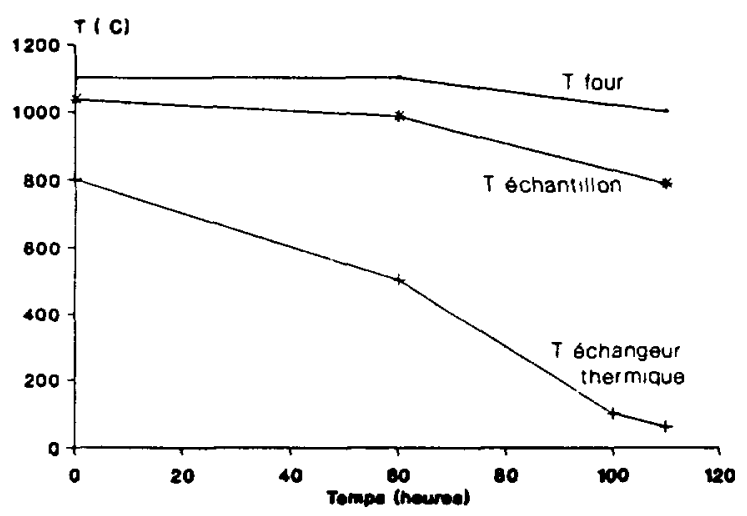

Fig. 2. - Evolutions thermiques lors du traitement de texturation par HEM.

[Thermal evolutions during texturing by HEM.]

\section{Résultats et discussion.}

Les lingots obtenus ont la forme du creuset $(4 \mathrm{~cm}$ de diamètre, $3 \mathrm{~cm}$ de haut). La forte réaction entre le liquide et le creuset d'alumine nécessite de détacher le lingot du creuset par découpe. La partie la plus proche du creuset est polluée (phases contenant de l'aluminium) et n'a pas été caractérisée. 
L'analyse par diffraction des rayons $X$ sur poudre de fragments de lingots broyés met en évidence la présence non seulement de la phase $\mathrm{YBa}_{2} \mathrm{Cu}_{3} \mathrm{O}_{7-1}$, mais aussi des phases $\mathrm{CuO}$, $\mathrm{BaCuO}_{2}$ et $\mathrm{Y}_{2} \mathrm{BaCuO}_{5}$ et montre ainsi la faible cinétique de la réaction péritectique.

Des échantillons découpés dans la partie basse centrale du lingot, parallèlement à la direction de solidification et présentant un plan de clivage, font apparaître uniquement les raies (001) caractéristiques de $\mathrm{YBa}_{2} \mathrm{Cu}_{3} \mathrm{O}_{7} \ldots$. Ceci révèle l'effet de texturation avec l'axe $c$ de $\mathrm{YBa}_{2} \mathrm{Cu}_{3} \mathrm{O}_{7-}$, perpendiculaire à la direction de solidification (parallèle au gradient thermique), en bon accord avec les autres techniques de texturation [3].

Il faut cependant noter que les plans de clivage ne sont pas parallèles entre eux au sein du matériau, bien qu'ils aient tous pour direction commune la direction de solidification.

L'observation par microscopie optique en lumière polarisée réfléchie d'un plan de clivage d'une dizaine de $\mathrm{mm}^{2}$ préalablement poli. met en évidence des domaines ferroélastiques confirmant que le plan observé est le plan basal (001) (Fig. 3). Elle permet également la visualisation de la phase verte $\mathrm{Y}_{2} \mathrm{BaCuO}_{5}$ sous forme d'inclusions.

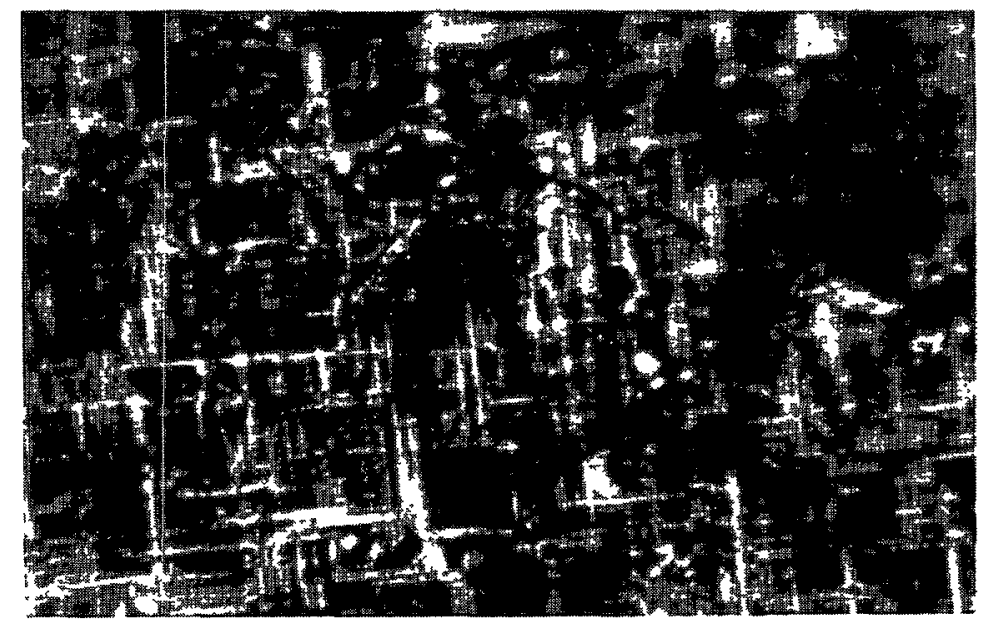

Fig. 3. - Domaines ferroélastiques observés par microscopie optique (X 320) sur un plan de clivage de $\mathrm{YBa}_{2} \mathrm{Cu}_{3} \mathrm{O}_{7}$.

[Ferroelastic domains observed by optical microscopy (X 320) on a $\mathrm{YBa}_{2} \mathrm{Cu}_{3} \mathrm{O}_{7-1}$ cleavage plane.]

Un plan de coupe traversant les plans $(a, b)$ et contenant la direction de croissance, observé par microscopie électronique à balayage (Fig. 4) fait apparaître la texturation avec des lamelles parallèles, larges d'environ $10 \mu \mathrm{m}$. Cependant on peut constater que l'échantillon est en fait constitué d'une juxtaposition de domaines texturés d'orientations différentes.

De nombreuses inclusions de phase verte $\mathrm{Y}_{2} \mathrm{BaCuO}_{5}$ réparties de façon uniforme apparaissent ; elles résultent d'une réaction incomplète entre $\mathrm{Y}_{2} \mathrm{BaCuO}_{5}$ et le liquide pour donner $\mathrm{YBa}_{2} \mathrm{Cu}_{3} \mathrm{O}_{7-1}$.

L'évolution de la résistivité électrique en fonction de la température sur des échantillons parallélépipédique $\left(2 \times 2 \times 8 \mathrm{~mm}^{3}\right)$, a été suivie par la méthode des quatre pointes.

Ils présentent une transition supraconductrice brutale $(\Delta T=1,8 \mathrm{~K})$ et une $T_{\mathrm{c}}$ onset de $92 \mathrm{~K}$.

La susceptibilité alternative d'un échantillon dont une face est un plan $(a, b)$ a été mesurée en appliquant le champ magnétique parallèlement puis perpendiculairement à l'axe $c$. Une 


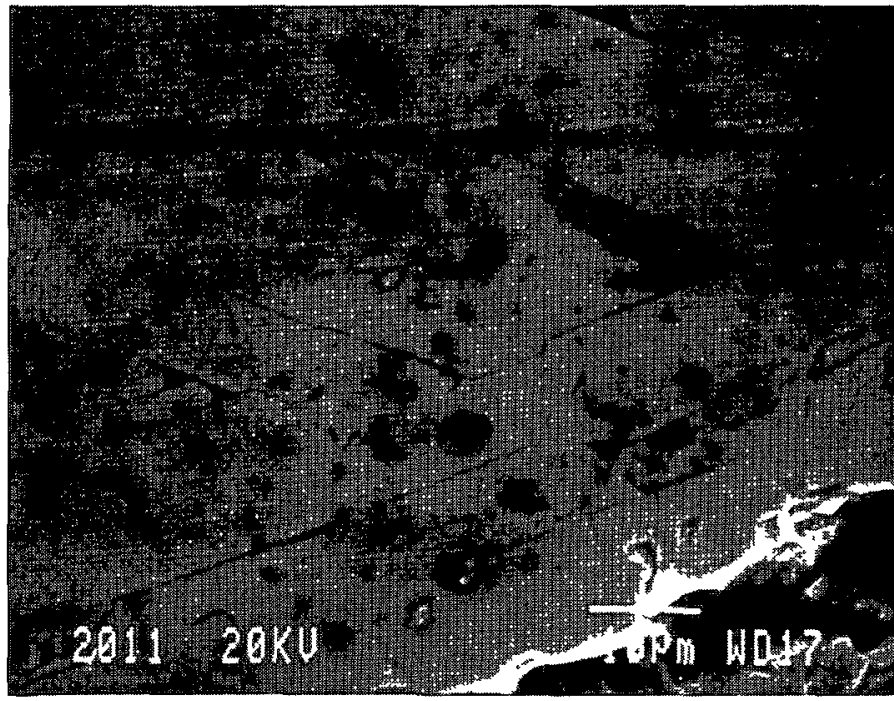

Fig. 4. - Domaines texturés fortement désorientés (X 1400).

[Textured domains highly disoriented (X 1400).]

anisotropie a ainsi été mise en évidence. A partir de ces mêmes mesures et sur la base du modèle de Bean [4], il est possible d'évaluer la densité de courant critique $J_{c}$; celle-ci, mesurée dans une direction parallèle aux plans $(a, b)$, s'élève à $5000 \mathrm{~A} / \mathrm{cm}^{2}$ à $77 \mathrm{~K}$, sans champ magnétique.

Les faibles valeurs de $J_{\mathrm{c}}$ de nos céramiques, comparées aux valeurs annoncées par d'autres auteurs peuvent s'expliquer par la désorientation entre deux grains (même si les axes $c$ de deux grains contigus sont parallèles) et par les nombreux défauts dans la céramique : porosité et fissuration importantes, présence de phases intergranulaires.

Une optimisation des paramètres d'élaboration, en particulier du traitement thermique de texturation, est en cours ; elle devrait améliorer sensiblement les propriétés des céramiques texturées obtenues par HEM.

\section{Conclusion.}

L'intérêt de l'utilisation de la méthode de l'échangeur thermique pour préparer des céramiques massives texturées de $\mathrm{YBa}_{2} \mathrm{Cu}_{3} \mathrm{O}_{7,}$ a été démontré. Sur les échantillons résultant de premiers essais, une densité de courant critique de $5000 \mathrm{~A} / \mathrm{cm}^{2}$ à $77 \mathrm{~K}$ sans champ magnétique a été mesurée.

L'amélioration de la méthode préparatoire de la céramique texturée devrait conduire à la réduction de la porosité et des fissurations et à une optimisation de la répartition de la phase verte afin d'augmenter les propriétés supraconductrices, et en particulier la densité de courant critique.

\section{Remerciements.}

Les auteurs tiennent à remercier Rhône-Poulenc Chimie pour l'aide matérielle apportée à la réalisation de ce travail. 


\section{Bibliographie}

[1] Khattak C. P. and Schmid F., Growth of large diameter crystals by HEM $^{\mathrm{TM}}$ for optical and laser applications, SPIE Advances in Optical Materials 505 (1984) 4-8.

[2] Murakami M. et al., Fine dispersion of $\mathrm{Y}_{2} \mathrm{BaCuO}_{5}$ inclusions through a peritectic reaction, International Worshop on Superconductivity co-sponsored by ISTEC and MRS (June 1992).

[3] Jin S. and Graebner J. E., Processing and fabrication techniques for bulk high$T_{\mathrm{c}}$ superconductors : a critical review, Mater. Sci. Engin. B 7 (1991) 243-260.

[4] Bean C. P., Rev. Mod. Phys. 36 (1964) 31. 